\title{
ESTIMACIÓN DEL NIVEL DE SERVICIO DE UN CARRIL LENTO
}

\author{
Víctor Gabriel Valencia Alaix \\ Profesor, Universidad Nacional de Colombia-Sede Medellín, Colombia. \\ Alfredo García \\ Profesor, HERG, Universitat Politècnica de València, España.
}

\section{RESUMEN}

La operación vehicular en una carretera convencional depende de la atención adecuada de los adelantamientos de los vehículos lentos por parte de los más rápidos; una alternativa es mediante la provisión de carriles auxiliares a lo largo de la vía antes de pasar a una carretera multicarril.

El objetivo es calibrar el modelo de simulación TWOPAS y aplicarlo en una carretera convencional española para estimar y comparar el nivel de servicio estimado en ella y en un carril lento con el resultado del procedimiento del Highway Capacity Manual - HCM.

Se observó la operación vehicular en una carretera convencional de España mediante el registro a través de cámaras de control dispuestas a lo largo de la carretera, y en el carril lento, de manera que sirvió para calibrar el modelo de microsimulación TWOPAS considerando parámetros operacionales del parque automotor y aplicándolo para estimar el nivel de servicio.

Se aplicó el procedimiento del HCM para estimar el nivel de servicio en segmentos de carretera con carril lento para comparar sus resultados con los obtenidos en la simulación.

El modelo de simulación usado fue el TWOPAS, inserto en el Traffic Analisys Module (TAM) del Interactive Highway Safety Design Model (IHSDM), cuyos resultados en términos de Percent Time Spent Following (PTSF), Average Travel Speed (ATS) y otros permite la evaluación operacional.

Los resultados y conclusiones permiten valorar la utilidad del modelo, la correspondencia de la realidad operacional en carretera y la norma española y la conveniencia operacional del carril lento estudiado. 


\section{INTRODUCCIÓN}

La calidad de la circulación y la seguridad en las carreteras convencionales está estrechamente relacionada con la facilidad de realizar los adelantamientos de lo contrario se manifiesta con reducción de la velocidad promedio, aumento de demoras, formación de grupos de vehículos, y dificultad de realizar maniobras seguras por lo que la rectificación del trazado y la dotación de carriles auxiliares son las soluciones típicas a implementar.

El mejor conocimiento del funcionamiento de los carriles auxiliares, como un carril lento o de ascenso, es el interés de este trabajo. Se buscar responder preguntas sobre la operación de estos carriles en condiciones de trazados generosos técnicamente con parque automotor de capacidad operacional alta y del comportamiento de indicadores que reflejan el efecto de estos carriles auxiliares.

Se han hecho estudios similares pero en condiciones de trazado pobres y parque automotor con baja capacidad operacional como el caso tratado por Valencia y García (2010) por lo que también se desea hacer comparaciones con los resultados de este trabajo.

\section{ESTADO DEL ARTE}

La provisión de carriles auxiliares a una carretera convencional considera algunas opciones según las condiciones de trazado y de tránsito que incluyen a los carriles de adelantamiento y de ascenso que son los fundamentos de las carreteras tipo $2+1$ sobre las cuales se consultó la literatura internacional.

Relacionado con los carriles de adelantamiento, Khan et al. (1991) estudiaron la manera de mejorar los adelantamientos y recomendaron que una metodología de evaluación debe considerar los mejoramientos operacionales para una longitud de influencia de la carretera. Tradicionalmente se ha reconocido que los carriles de adelantamiento y de ascenso son soluciones apropiadas para carreteras con volúmenes bajos y medios para los cuales no se justifica una carretera de cuatro carriles.

En cuanto a las medidas de efectividad de carriles de adelantamiento Khan et al. (1991) definieron una metodología para estimar el efecto en los niveles de servicio que produce la implementación de carriles de adelantamiento. De los indicadores considerados, como por ejemplo, porcentaje de vehículos en grupo y tamaño del grupo, oportunidades de adelantamiento seguras ofrecidas para niveles de flujo dados, tasa de adelantamientos por sección, y porcentaje de oportunidades de adelantamiento (adelantamientos/veh-km), se decidió, el uso del porcentaje de vehículos en grupo y la velocidad promedio. La evaluación operacional de la gran variedad de configuraciones de carretera con y sin 
carriles de adelantamiento se realizó con modelos de simulación para establecer funciones que relacionan las características principales de la carretera y el desempeño del tránsito en términos los indicadores seleccionados.

Por su parte Messer (1983) encontró que el Porcentaje de Tiempo Consumido en el Seguimiento (PTSF) formando grupos de vehículos a lo largo de un tramo de carretera puede estimarse con la medida del porcentaje de vehículos que circulan formando grupos con intervalos menores a cinco segundos; el PTSF se prefiere sobre la velocidad porque es más sensible al volumen de tránsito pero más difícil de medir.

May (1991) utilizó como criterio para medir el PTSF cuatro segundos de intervalo y el Manual de aplicación del modelo de simulación del tránsito TWOPAS que es el corazón del Traffic Analisys Module (TAM) dentro del Interactive Highway Safety Design Model (IHSDM) sugiere que se use tres segundos (FHWA, 2013).

Los carriles de adelantamiento mejoran la operación del tránsito en términos de reducción de los grupos de vehículos y de aumento de velocidad después del carril a lo largo de una distancia denominada longitud efectiva que depende del nivel de volumen de tránsito.

Khan et al. (1991) mediante simulaciones, desarrollaron pautas para determinar la longitud del carril de adelantamiento apropiada y su distancia efectiva posterior a él.

En un estudio del Instituto de Investigaciones de Midwest, Harwood y St. John (1986) concluyeron que la reducción del porcentaje de agrupamiento puede persistir sobre una longitud efectiva de 8 a 12,8 kilómetros, dependiendo de la longitud del carril de adelantamiento, el flujo de tránsito, su composición y de la disponibilidad de oportunidades de adelantamiento más adelante en la vía.

Morrall y Thomson (1990) consideraron que las medidas como el porcentaje de tiempo gastado en el seguimiento y la oportunidad de sobrepaso, además de ser más significativas en términos de nivel de servicio percibido por el conductor, son medidas que pueden ser usadas para evaluar la efectividad de carriles de adelantamiento.

May (1991) observó la operación del tránsito en cinco carreteras de California caracterizándola mediante indicadores como la velocidad y el PTSF. Se encontraron beneficios operacionales medidos con PTSF, el porcentaje del tránsito con intervalos de dos segundos y los vehículos solitarios. Todas estas medidas de efectividad indicaron mejoras significativas y las mayores de todos los sitios estudiados fueron para el sitio en terreno montañoso y carriles largos. Se prefirieron aquellas medidas que podrían ser expresadas como una función de la longitud del carril de adelantamiento, por ejemplo, el número de adelantamientos por su longitud, pues permite comparar varias opciones de longitudes. 
Al Kaisy y Freedman (2010) realizaron mediciones en carriles de adelantamiento, de dos sitios de Montana, de los beneficios en lugares situados antes, durante y después del carril auxiliar, usando como indicadores operacionales, además del PTSF y la velocidad promedio de recorrido (ATS), otros indicadores como porcentaje de perseguidores, densidad de perseguidores y relación entre la velocidad y la velocidad a flujo libre (ATS/FFS). Encontraron que efectivamente se presentaron los beneficios en la longitud efectiva, en una magnitud que se correspondía con los valores indicados en el HCM (TRB, 2010).

En 2013, Freedman y Al-Kaisy observaron la formación de grupos de vehículos y de la realización de las maniobras de adelantamiento dentro de un carril de adelantamiento en la Carretera 287 de EEUU y midieron la división del volumen, porcentaje de perseguidores y densidad de perseguidores por carril, que mostraron que la actividad de adelantamientos se realiza en la primera mitad de milla del carril de adelantamiento, lo cual puede sugerir otro criterio de la longitud optima de estos carriles auxiliares.

Cuando se refiere a pautas para el diseño de carriles de adelantamiento Morrall y Thomson (1990) capitalizaron la experiencia obtenida a través del estudio de carriles experimentales y en otro trabajo de planificación y diseño de carriles de adelantamiento en la carretera Transcanadiense estableciendo criterios para definir el lugar y para evaluar las opciones de carriles de adelantamiento como los siguientes:

a) Minimizar los costos de construcción.

b) El lugar debe ser lógico para el conductor por las necesidades de adelantamientos importantes.

c) Evitar secciones de carretera con especificaciones de diseño reducidas.

d) Disponer distancias de visibilidad adecuadas al principio y al final del carril de adelantamiento.

e) Evitar intersecciones o puntos de acceso de uso diario en el inicio o final del carril de adelantamiento.

f) Evitar restricciones físicas que afecte la continuidad de la berma.

g) Considerar que los carriles de adelantamiento localizados inmediatamente antes o después de tramos de cuatro carriles no son efectivos.

En relación a los carriles de ascenso o lentos, Morrall y Thomson (1990) consideraron que en pendientes largas y escarpadas pueden ser más largos que lo recomendado en condiciones normales para los carriles de adelantamiento, a causa de los grandes diferenciales de velocidad entre automóviles y camiones.

Para la evaluación del comportamiento operacional en estos carriles Glennon (1970) estudió las velocidades de camiones y su relación con el índice de accidentalidad y halló que dicho indicador se incrementa significativamente cuando la reducción de velocidad 
de los camiones respecto al resto del tránsito supera los $15 \mathrm{~km} / \mathrm{h}$. Este resultado ha sido acogido por muchos autores y entidades oficiales de carreteras para construir criterios para la justificación de carriles de ascenso con el objetivo de la seguridad.

Para evaluar la reducción de velocidad, los diseñadores recomiendan como referente el uso de un camión tipo con una relación peso/potencia que lo represente (Khan et al., 1990).

El HCM (TRB, 2010) y el Libro Verde de la AASHTO (2011) consideran en los criterios de justificación de carriles lentos camiones con relación peso/potencia de 90 kg/CV.

Valencia, Bedoya y Osorno (1996) midieron relaciones peso/potencia neta de vehículos pesados en carreteras colombianas con base en ecuaciones de equilibrio dinámico en pendientes ascendentes y concluyeron que su valor promedio ponderado, según composición vehicular, es de $157 \mathrm{~kg} / \mathrm{CV}$ que es más alto que los referidos en los criterios de justificación de carriles auxiliares en las referencias internacionales, como el HCM; concluyéndose que los camiones colombianos tienen capacidades operacionales mucho menores motivando la creación de justificaciones de carriles de ascenso correspondientes a las condiciones del país.

El HCM (TRB, 2010) propone una metodología para estimar el efecto operacional que produce la presencia de un carril de adelantamiento en un segmento direccional de carretera convencional.

El efecto de un carril de adelantamiento en el PTSF es reducirlo a lo largo de una distancia más adelante del carril que va aumentando al nivel de una carretera sin carril de adelantamiento; esta longitud efectiva según el HCM (TRB, 2010) también se refleja en el comportamiento de la ATS. Los pasos a seguir serían:

1: Realizar el análisis operacional en la carretera sin carril de adelantamiento.

2: Dividir el segmento en regiones.

$\mathrm{L}_{\mathrm{u}}=$ Longitud antes del carril de adelantamiento.

$\mathrm{L}_{\mathrm{pl}}=$ Longitud del carril de adelantamiento.

$\mathrm{L}_{\mathrm{de}}=$ longitud efectiva.

$\mathrm{L}_{\mathrm{d}}=$ Longitud después de la longitud efectiva $\mathrm{L}_{\mathrm{de}}$.

3: Determinar el PTSF para todo el segmento de carretera de análisis afectada por el carril de adelantamiento y está dado por la Ecuación (1): 


$$
\operatorname{PTSF}_{p l}=\frac{\operatorname{PTSF}_{d}\left\lfloor L_{u}+L_{d}+f_{p l, P T S F} L_{p l}+\left(\frac{1+f_{p l, P T S F}}{2}\right) L_{d e}\right\rfloor}{L_{t}}
$$

$\mathrm{PTSF}_{p l}=$ PTSF para el segmento de carretera afectado por el carril de adelantamiento (decimal).

$f_{p l, P T S F}=$ Factor de corrección por el efecto del carril de adelantamiento en el PTSF.

El valor de PTSF en el carril de adelantamiento está entre 0,58 y 0,62 del valor del PTSF en la región anterior con longitud $\mathrm{L}_{\mathrm{u}}$.

4: Determinar la Velocidad Media de recorrido ATS para todo el segmento de carretera de análisis está afectado por el carril de adelantamiento y está dado por la Ecuación (2):

$$
A T S_{p l}=\frac{A T S_{d} L_{t}}{L_{u}+L_{d}+\left(\frac{L_{p l}}{f_{p l, A T S}}\right)+\left(\frac{2 L_{d e}}{1+f_{p l, A T S}}\right)}
$$

$\mathrm{ATS}_{p l}=$ ATS para el segmento de carretera afectado por el carril de adelantamiento (decimal).

$f_{p l, A T S}=$ Factor de corrección por el efecto del carril de adelantamiento en el ATS .

El valor de ATS en el carril de adelantamiento está entre $8 \%$ y $11 \%$ más alto que el valor del ATS en la región anterior con longitud $\mathrm{L}_{\mathrm{u}}$.

5: Determinar el Nivel de Servicio según la Tabla 1.

\begin{tabular}{|c|c|c|c|c|}
\hline \multirow{2}{*}{ LOS } & \multicolumn{2}{|c|}{ Class I Highways } & $\begin{array}{c}\text { Class II } \\
\text { Highways }\end{array}$ & $\begin{array}{c}\text { Class III } \\
\text { Highways }\end{array}$ \\
\cline { 2 - 5 } & ATS (mi/h) & PTSF (\%) & $\begin{array}{c}\text { PTSF (\%) } \\
\text { PFFS (\%) }\end{array}$ \\
\hline $\mathrm{A}$ & $>55$ & $\leq 35$ & $\leq 40$ & $>91.7$ \\
\hline $\mathrm{B}$ & $>50-55$ & $>35-50$ & $>40-55$ & $>83.3-91.7$ \\
\hline $\mathrm{C}$ & $>45-50$ & $>50-65$ & $>55-70$ & $>75.0-83.3$ \\
\hline $\mathrm{D}$ & $>40-45$ & $>65-80$ & $>70-85$ & $>66.7-75.0$ \\
\hline $\mathrm{E}$ & $\leq 40$ & $>80$ & $>85$ & $\leq 66.7$ \\
\hline
\end{tabular}

Tabla 1. Nivel de Servicio para carreteras convencionales considerando autos, TRB (2010).

El análisis operacional del efecto de un carril de ascenso en una carretera convencional se realiza con el mismo procedimiento descrito para carriles de adelantamiento pero con tres diferencias importantes:

- Los factores de corrección por el efecto de un carril de ascenso son otros. 
- El análisis sin carril de ascenso se realiza con el procedimiento normal para una carretera sin carril de ascenso, pero en el caso de una pendiente ascendente específica.

- Las longitudes $\mathrm{L}_{\mathrm{u}} \mathrm{y} \mathrm{L}_{\mathrm{d}}$ se establecen en cero.

El HCM (TRB, 2010) y el Libro Verde de la AASHTO (2011) justifican la dotación de un carril de ascenso si cumplen las condiciones:

1. El flujo de tránsito en la pendiente ascendente exceda los $200 \mathrm{veh} / \mathrm{h}$.

2. El flujo de camiones en la pendiente exceda los 20 camiones/h.

3. Y una de las siguientes:

a. Se cuenta con una reducción de velocidad de $15 \mathrm{~km} / \mathrm{h}$ o más para el camión típico de $120 \mathrm{~kg} / \mathrm{kW}$ (200lb/hp ó $90 \mathrm{~kg} / \mathrm{CV})$.

b. Se presenta un nivel de servicio E o F en la pendiente.

c. Se experimenta una reducción de dos o más niveles de servicio cuando se circula entre el acceso y la pendiente.

Según AASHTO (2011), el concepto de carreteras 2+1 ha mejorado la eficiencia operacional y reducida las colisiones en carreteras convencionales seleccionadas. Consiste en suministrar una sección transversal de tres carriles continua acomodando a lo largo de la carretera carriles de adelantamiento de manera alterna a cada dirección de circulación.

Puede ser un tratamiento adecuado para carreteras con volúmenes más altos que aquellos que pueden atender un solo carril de adelantamiento, pero no tan altos como para justificar una carretera de cuatro carriles. Podría operar generalmente, al menos, dos niveles de servicio más altos que una carretera convencional atendiendo el mismo volumen de tránsito.

Un estudio realizado por el Instituto de Transporte de Texas (2001) consideró una serie de simulaciones realizadas con el TWOPAS en una carretera hipotética variando condiciones de tránsito como volumen bidireccional, porcentaje de camiones, longitud de carriles y espaciamiento entre ellos dispuestos emparejados a cada lado de una carretera de dos carriles. Se alcanzaron resultados en términos del PTSF y determinó criterios de diseño de estos carriles denominados carreteras "Super 2".

Brewer y Venglar (2010) analizaron el mejoramiento de la operación vehicular en carreteras "Super 2" como alternativa de bajo costo antes de una carretera con 4 carriles.

De acuerdo a la normativa para el trazado de carreteras convencionales de España Norma 3.1-IC. Trazado (Orden FOM/273/2016 de 19 de febrero de 2016) (MINISTERIO DE FOMENTO, 2016) el establecimiento de un carril adicional en rampa en una carretera convencional considera el mismo criterio del Libro Verde (AASHTO, 2011) y del HCM 
(TRB, 2010) que exige el cumplimiento simultáneo de las tres condiciones indicadas anteriormente.

Para definir el inicio y final de un carril adicional en rampa, y consecuentemente su longitud, se tendrá en cuenta:

- El inicio del carril adicional se situará donde los vehículos pesados reduzcan su velocidad en diez kilómetros por hora $(10 \mathrm{~km} / \mathrm{h})$ respecto de la velocidad de proyecto $(\mathrm{Vp})$, sin considerar velocidades iniciales mayores que cien kilómetros por hora $(\ngtr 100 \mathrm{~km} / \mathrm{h}$ ). La estimación de la reducción de velocidad en las rampas se podrá hacer de acuerdo con los perfiles de velocidad que contempla la norma.

- El final del carril adicional se situará donde, una vez superado el vértice del acuerdo, el vehículo pesado recupere la velocidad correspondiente que hizo necesario el establecimiento del carril adicional y, en el caso de carreteras convencionales, donde además se disponga de visibilidad de adelantamiento para la velocidad de proyecto $(\mathrm{Vp})$.

Respecto a modelos de simulación se comentará sobre el TWOPAS usado en este caso, el cual fue desarrollado por el Midwest Research Institute para la Federal Highway Administration (FHWA), que simula la operación del tránsito en carreteras de dos carriles (con o sin carriles auxiliares) mediante la revisión de la posición, velocidad y aceleración/deceleración y el avance de cada vehículo en la carretera a intervalos de un segundo.

TWOPAS permite la simulación detallada, basada en las características del tránsito y de la carretera especificadas por el usuario, tales como geometría de las vías, restricciones de adelantamiento, presencia de carriles auxiliares, curvatura vertical u horizontal, control del tránsito o variables que influyen sobre la distancia de visibilidad.

El modelo incorpora decisiones de adelantamiento realísticas y rechazos del adelantamiento. También puede simular las operaciones del tránsito en carriles de adelantamiento y de ascenso, incluyendo la operación de la adición y de áreas de transición de anchura de carriles y en los cambios de carril dentro de secciones de carriles de adelantamiento o de ascenso.

Según el estudio realizado por la NCHRP (1998), TWOPAS fue utilizado para desarrollar el capítulo sobre capacidad y niveles de servicio en carreteras de dos carriles del HCM considerando como capacidad operativa del parque automotor de los EEUU los valores que se encuentran por omisión en el archivo de configuración del TAM, modulo del IHSDM (FHWA, 2013). 


\section{OBJETIVOS}

\subsection{General}

Calibrar un modelo de microsimulación y aplicarlo en la evaluación de un carril lento en España para determinar el nivel de servicio y el efecto de un enlace en su operación.

\subsection{Específicos}

- Observar la operación del tránsito en una carretera convencional con carril lento en España.

- Calibrar el modelo TWOPAS para las condiciones observadas.

- Aplicar el TWOPAS para evaluar operacionalmente la carretera y el carril lento.

- Determinar el Nivel de servicio con la metodología del HCM y con la simulación.

\section{METOdología}

\subsection{Tramo experimental}

La vía observada es una carretera convencional, denominada CV-13, de la Comunidad Valenciana con una longitud de 16,500 km distribuidos en una autovía (P.K. 0 a 2,500) y la primera calzada de la futura autovía que opera como una carretera convencional (P.K. 2,500 a 16,500) (GENERALITAT VALENCIANA, 2010). Está desde las proximidades de Benlloch hacia la costa (Torreblanca), pasando por el aeropuerto de Castellón y afectando a los municipios de Vilanova d'Alcolea y Alcala de Xivert como muestra el esquema de la Figura 1. Hay otras estructuras en cruces a distinto nivel como el paso por debajo de la AP-7 y las conexiones de la CV-13 con las infraestructuras existentes que conducen a las poblaciones mencionadas.

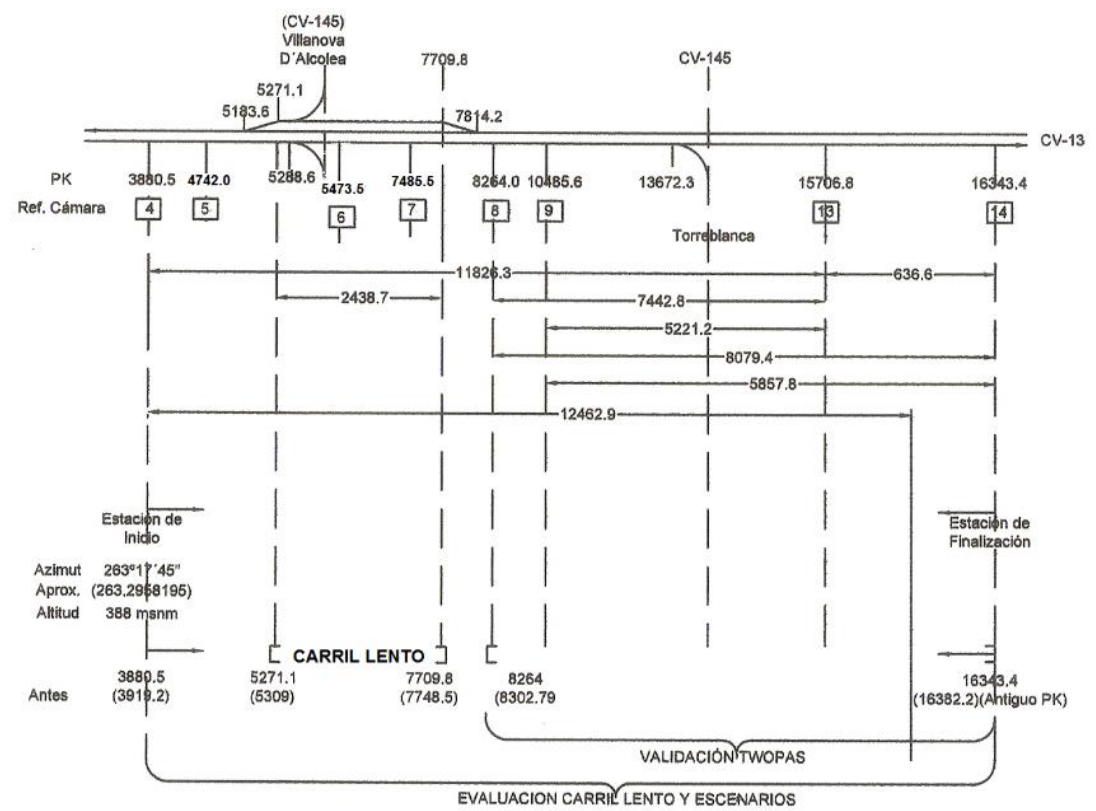

Figura 1. Ubicación del tramo de la validación del TWOPAS en la CV-13. 
La sección de la carretera está compuesta por dos carriles de 3,50 m con arcenes asimétricos de 1,50 metros y 2,50 metros, a la derecha e izquierda de la vía en el sentido de P.K. crecientes, con bermas de 1,00 m, resultando en una anchura total de 13,00 m. La carretera se proyectó como una autovía A-100, con capacidad de tráfico de 50.000 veh/día y un tráfico pesado T0.

El perfil longitudinal de la carretera tiene pendientes y rampas próximas al 5\% lo que ha originado un carril adicional para vehículos lentos de $2700 \mathrm{~m}$ de longitud.

Con el objetivo de mejorar la seguridad vial se ha dotado de una instalación ITS (Sistemas Inteligentes de Transporte) de vigilancia y control convencional integrado por 14 cámaras que ha servido para monitorizar por imágenes el 100\% de la carretera, lo cual ha sido útil en la toma de información de tránsito.

Otras especificaciones de la carretera son:

- Velocidad específica del tronco: $100 \mathrm{~km} / \mathrm{h}$.

- Radio mínimo: $450 \mathrm{~m}$.

- Número de carriles:

- P.K. 3+200 al P.K. 16+500: Calzada única de 2 carriles.

- P.K. 5+200 al P.K. 7+900: Calzada única de 3 carriles (con carril adicional para vehículos lentos).

En las Figura 2 se muestra el entorno en el que discurre la carretera CV-13.

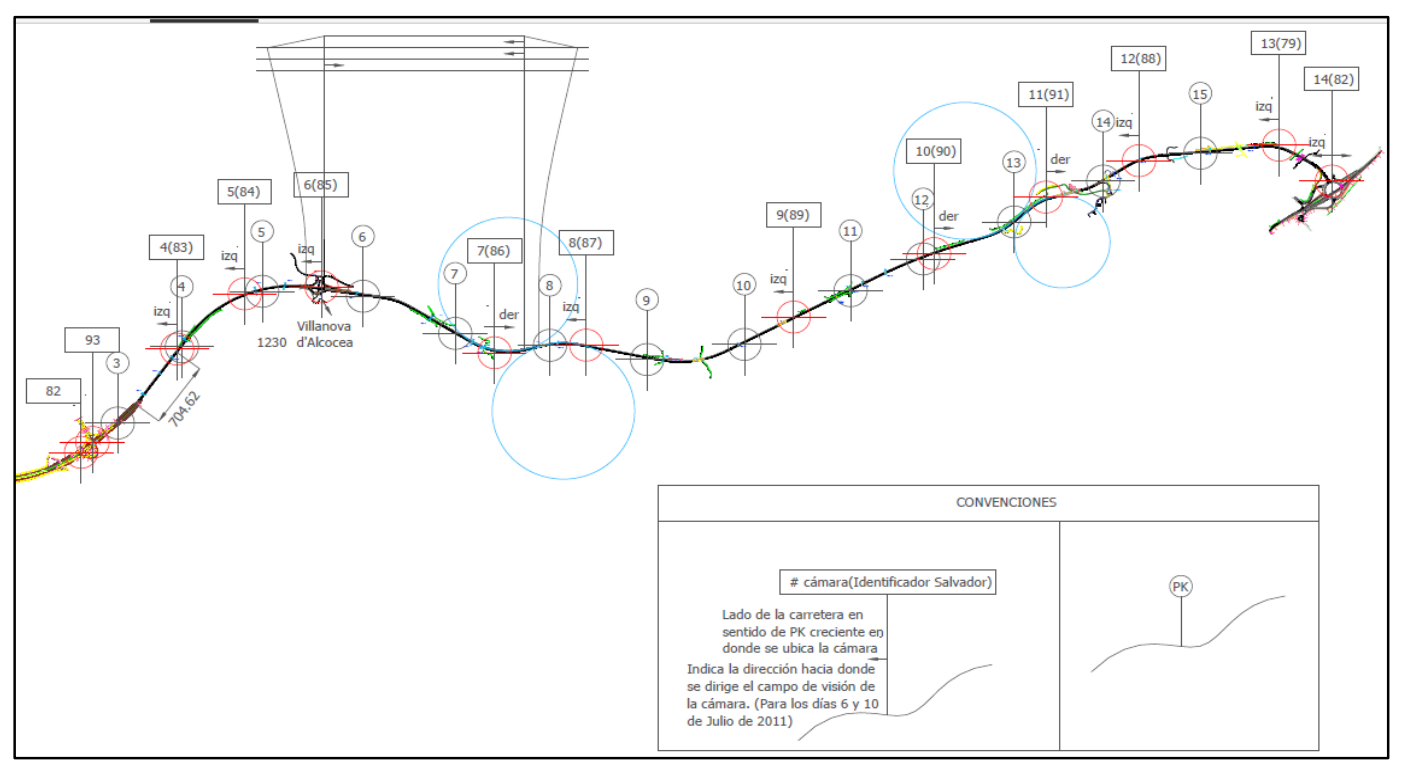

Figura 2. Esquema general del trazado en planta de la CV-13.

El volumen medido para el sentido creciente de P.K. es de $184 \mathrm{veh} / \mathrm{h}$ y en el inverso 191 $\mathrm{veh} / \mathrm{h}$. 
En cada cámara se observó el tránsito y se midió:

- Subtramos de carretera, entre cámaras, en los cuales los vehículos viajaban en estado de seguimiento y consecuentemente el tiempo de seguimiento.

- Velocidad de recorrido (ATS).

- Tiempo de recorrido

- Porcentaje de tiempo consumido en el seguimiento o demora (PTSF)

- Volumen horario y composición vehicular.

- Estimación de la velocidad deseada en tramos horizontales como la velocidad a flujo libre.

- Velocidad máxima de entrada a la carretera, por cada tipo de vehículo.

Con toda la información obtenida se pudo determinar las condiciones de geometría de la vía y de tránsito del carril lento.

\subsection{Calibración del TWOPAS}

Con la información obtenida a partir de los datos observados en la CV13 y descritos en el aparte anterior se caracteriza el comportamiento actual de la vía y se estiman los valores de indicadores operacionales para calibrar el TWOPAS para España; Valencia (2016) calibró el TWOPAS para Colombia y la metodología aplicada es la que se considera en este trabajo.

\subsection{Estimación del nivel de servicio según el HCM}

El HCM de 2010 propone un método para estimar el nivel de servicio en tramos de carreteras de dos carriles conteniendo un carril lento mediante el valor promedio ponderado del PTSF y de la ATS y según un modelo de comportamiento de estas dos variables el cual se aplica a la CV13.

\subsection{Aplicación del TWOPAS calibrado en la CV13}

Se aplica el TWOPAS calibrado en la CV13 para conocer el PTSF y ATS, entre otros indicadores operacionales, a lo largo de toda la carretera y especialmente en el carril lento y poder estimar su nivel de servicio. Se hace una comparación con los resultados obtenidos con el método del HCM y obtener conclusiones sobre su aplicabilidad.

\section{RESULTADOS}

\subsection{Calibración}

Para representar el comportamiento de la carretera se configuró el TAM mediante el uso del programa ADMINISTRATION TOOL en el archivo CONFIGURACION6. Aquí se modificaron algunos parámetros del modelo de simulación entre los que se destacan las características de prestación de los vehículos: 
- Tipo de vehículo.

- Relación peso/potencia. Ver Tabla 2.

- Relación Área frontal proyectada/peso.

- Longitud.

- Factor de corrección de la potencia.

- Factor de corrección del arrastre aerodinámico.

- Velocidad máxima de entrada para el sentido 1 de circulación en la carretera.

- Velocidad máxima de entrada para el sentido 2 de circulación en la carretera.

- Porcentaje de participación de este tipo de vehículo en el sentido 1 de circulación en la carretera de acuerdo a la categoría que le corresponde.

- Porcentaje de participación de este tipo de vehículo en el sentido 2 de circulación en la carretera de acuerdo a la categoría que le corresponde.

\subsubsection{Velocidades residuales de vehículos pesados}

En la CV13 se estimó la relación peso/potencia usada por los camiones aplicando una expresión que relaciona las fuerzas y resistencias que participan en el desplazamiento de un camión al circular por una rampa a velocidad residual; para ello se midió esta velocidad en una rampa del 4,7 \% ubicada al lado de la cámara de control 11 en el P.K. 13+470,900. En la Tabla 2 se presentan los resultados por tipo de camión que considera el TWOPAS definidos de acuerdo al parque automotor observado en la CV-13. Este valor se usó en la aplicación del TWOPAS para su calibración.

\begin{tabular}{|c|c|c|c|c|}
\hline $\begin{array}{l}\text { TIPO DE CAMIÓN } \\
\text { PARA TWOPAS }\end{array}$ & \begin{tabular}{|c|} 
Descripción para \\
observación en vídeos
\end{tabular} & $\begin{array}{c}\text { VELOCIDAD } \\
\text { RESIDUAL } \\
(\mathbf{k m} / \mathbf{h}) \\
\end{array}$ & $\begin{array}{c}\text { DESVIACIÓN } \\
\text { ESTÁNDAR } \\
(\mathbf{k m} / \mathbf{h}) \\
\end{array}$ & \begin{tabular}{|c|} 
RELACIÓN \\
PESO/POTENCIA \\
ESTIMADA \\
(kg/CV) \\
\end{tabular} \\
\hline 1 & C art & 42.5 & 9.1 & 85.7 \\
\hline 2 & \begin{tabular}{|c|} 
C rigido2 (Contenedor)+ \\
Cart1 (Rigido + \\
remolque)
\end{tabular} & 41.6 & N.D. & 88.0 \\
\hline 3 & $\mathrm{C}$ rigido $+\mathrm{C}$ rigidovacio & 40.4 & 3.4 & 90.6 \\
\hline 4 & $\begin{array}{c}\text { C rigidopeque (Volquete, } \\
\text { grúa) }\end{array}$ & 45.8 & 13.7 & 82.7 \\
\hline
\end{tabular}

Tabla 2. Velocidad residual y relación peso/potencia de camiones en la CV-13. (Valencia, 2016)

Se ejecutó el TWOPAS y sus resultados son entregados por el modelo en las estaciones o P.K. a lo largo de la carretera y por tramos con características especifica como el tramo de solo carretera convencional y el tamo que contiene el carril lento.

Algunas características de la aplicación del TWOPAS se indican a continuación:

IHSDM Version: v9.1.0 (Feb 09, 2014)

Traffic Analysis Module: v1.4.0 (Apr 26, 2010)

Project Title: VALIDACIÓN TWOPAS 2 
Project Comment: Created Mon Jul 01 17:34:45 CEST 2013

Highway Title: cv-13 prueba

Highway Comment: Created Thu Jul 04 12:46:45 CEST 2013

Highway Version: 8

Evaluation Title: EVALUACIÓN DE LA CARRETERA CV-13

Evaluation Comment: Created Sat Sep 05 15:56:39 COT 2015

Minimum Station: $3+880.500$

Maximum Station: $16+343.400$

Configuration Name: CONFIGURACION6

En la Tabla 3 se presenta una réplica de los datos de entrada generales.

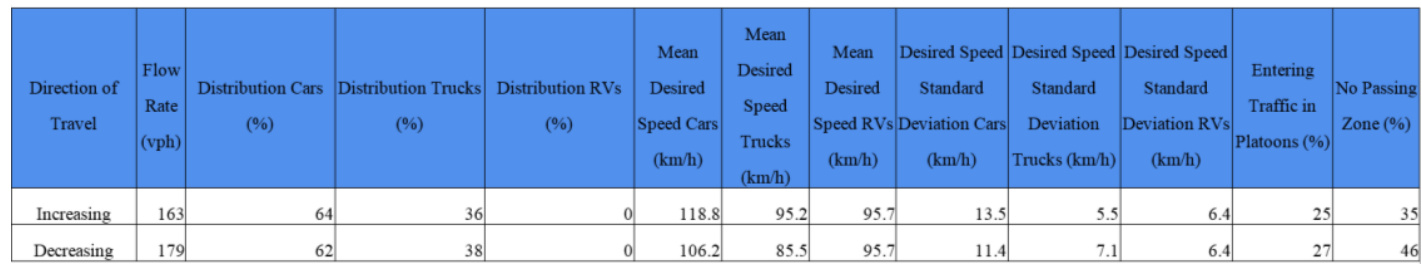

\section{Tabla 3. Datos de entrada del tránsito.}

La carretera CV-13 que se consideró para hacer la calibración del TWOPAS está comprendido entre el P.K. 8+264,000 y P.K.16+333,400; en la Tabla 4 se presentan los resultados de la simulación.

\begin{tabular}{|c|c|c|c|c|c|c|c|c|c|c|}
\hline $\begin{array}{c}\text { Direction of } \\
\text { Travel }\end{array}$ & $\begin{array}{c}\text { Flow Rate from } \\
\text { Simulation } \\
\text { (vph) }\end{array}$ & $\begin{array}{c}\text { Percent Time } \\
\text { Spent } \\
\text { Following (\%) }\end{array}$ & $\begin{array}{c}\text { Average } \\
\text { Travel } \\
\text { Speed } \\
(\mathrm{km} / \mathrm{h}) \\
\end{array}$ & $\begin{array}{c}\text { Trip } \\
\text { Time } \\
(\mathrm{min} / \mathrm{veh})\end{array}$ & $\begin{array}{c}\text { Traffic } \\
\text { Delay } \\
\text { (min/veh) }\end{array}$ & $\begin{array}{c}\text { Geometric } \\
\text { Delay } \\
(\mathrm{min} / \mathrm{veh})\end{array}$ & $\begin{array}{c}\text { Total } \\
\text { Delay } \\
(\mathrm{min} / \mathrm{veh})\end{array}$ & $\begin{array}{l}\text { Number } \\
\text { of Passes }\end{array}$ & $\begin{array}{c}\text { Distance } \\
\text { Traveled } \\
(\mathrm{km})\end{array}$ & $\begin{array}{c}\text { Total } \\
\text { Travel } \\
\text { Time } \\
\text { (veh-hrs) } \\
\end{array}$ \\
\hline Increasing & 162 & 48 & 87.2 & 5.5 & 0.4 & 0.5 & 0.9 & 47 & $1,304.0$ & 14.9 \\
\hline Decreasing & 180 & 44 & 65.2 & 7.4 & 0.7 & 2.0 & 2.8 & 113 & $1,446.4$ & 22.2 \\
\hline Combined & 342 & 46 & 75.6 & 6.5 & 0.6 & 1.3 & 1.9 & 160 & $2,750.4$ & 37.1 \\
\hline
\end{tabular}

Tabla 4. Resultados entre P.K. 8+264,000 hasta P.K.16+333,400 de solo carretera convencional.

Para la calibración del TWOPAS (TAM) se analiza el grado de aproximación entre los valores de indicadores operacionales simulados por el modelo y los valores correspondientes medidos directamente por observaciones del tránsito en un tramo de la carretera CV-13 comprendido entre el P.K. 8+264,000 hasta P.K.16+333,400; los resultados se presentan en la Tabla 5.

Los valores de la columna titulada Grado de Aproximación corresponde al porcentaje por exceso o defecto que el valor del indicador simulado en la operación vehicular en la carretera, por lo tanto, un valor negativo indica que TWOPAS subestima el valor medido en la vía. Por su parte, el número del grado de aproximación refleja la proximidad de la anterior estimación y de acuerdo a los niveles de aproximación que se ha observado en 
calibraciones y validaciones con el uso modelos de microsimulación resulta aceptable si este valor fuese menor de 10; por lo tanto, puede considerarse que, aunque los resultados están ligeramente superior a este valor referente, el TWOPAS estaría calibrado a las condiciones de una carretera como la CV-13 en condiciones de España.

\begin{tabular}{|c|c|c|c|c|c|c|}
\hline \multirow[b]{2}{*}{ INDICADOR OPERACIONAL } & \multicolumn{2}{|c|}{ MEDIDOS EN LA CV13 } & \multicolumn{2}{|c|}{$\begin{array}{l}\text { SIMULADOS CON } \\
\text { TWOPAS (TAM) }\end{array}$} & \multicolumn{2}{|c|}{$\begin{array}{c}\text { GRADO DE } \\
\text { APROXIMACIÓN (\%) }\end{array}$} \\
\hline & $\begin{array}{l}\text { Dirección 1 } \\
\text { (Pks } \\
\text { crecientes) }\end{array}$ & $\begin{array}{c}\text { Dirección } 2 \\
\text { (Pks } \\
\text { decrecientes) }\end{array}$ & $\begin{array}{c}\text { Dirección 1 } \\
\text { (Pks } \\
\text { crecientes) }\end{array}$ & $\begin{array}{c}\text { Dirección } 2 \\
\text { (Pks } \\
\text { decrecientes) }\end{array}$ & $\begin{array}{c}\text { Dirección 1 } \\
\text { (Pks } \\
\text { crecientes) }\end{array}$ & $\begin{array}{c}\text { Dirección } 2 \\
\text { (Pks } \\
\text { decrecientes) }\end{array}$ \\
\hline FLUJO (veh/h) & 181,8 & 178,7 & 162 & 180 & $-10,9 \%$ & $0,7 \%$ \\
\hline PTSF (\%) & 36 & 39 & 48 & 44 & $33,3 \%$ & $12,8 \%$ \\
\hline $\begin{array}{c}\text { VEOCIDAD PROMEDIO DE } \\
\text { RECORRIDO }(\mathrm{km} / \mathrm{h})\end{array}$ & 93,1 & 81,9 & 87,2 & 65,2 & $-6,3 \%$ & $-20,4 \%$ \\
\hline $\begin{array}{c}\text { TIEMPO DE RECORRIDO } \\
(\mathrm{m} \text { in/ve } h)\end{array}$ & 5,17 & 6,1 & 5,5 & 7,4 & $6,4 \%$ & $21,3 \%$ \\
\hline $\begin{array}{c}\text { NUMERO DE } \\
\text { ADELANTAMIENTOS }\end{array}$ & ND & ND & 47 & 113 & ND & ND \\
\hline $\begin{array}{l}\text { DISTANCIA RECORRIDA } \\
\text { TOTAL }(\mathrm{km})\end{array}$ & 1421,19 & $1.452,55$ & 1304 & 1446,4 & $-8,2 \%$ & $-0,4 \%$ \\
\hline $\begin{array}{c}\text { TIEMPO TOTAL DE VIAJE } \\
\text { (veh-h) }\end{array}$ & 15,1 & 19 & 14,9 & 22,2 & $-1,3 \%$ & $16,8 \%$ \\
\hline \multicolumn{5}{|c|}{ PROMEDIO DEL GRADO DE APROXIM ACION } & $2,2 \%$ & $5,1 \%$ \\
\hline \multicolumn{5}{|c|}{ PROMEDIO DE LOS VALORES ABSOLUTOS DEL GRADO DE APROXIM ACION } & $11,1 \%$ & $12,1 \%$ \\
\hline
\end{tabular}

Tabla 5. Grados de aproximación entre valores medidos en la CV-13 y simulados con TWOPAS.

\subsection{Nivel de servicio con HCM}

Al aplicar la metodología para estimar el nivel de servicio del HCM (TRB, 2010) en el carril lento con las siguientes características:

- Volumen direccional en ascenso $=179 \mathrm{veh} / \mathrm{h}$

- Volumen direccional en descenso $=163 \mathrm{veh} / \mathrm{h}$

- Distribución direccional: $52 \%$ en el ascenso y $48 \%$ en el descenso.

- $\mathrm{FHP}=0,90$

- Zonas de adelantamiento prohibido en las dos direcciones $=62,8 \%$

- Porcentaje de camiones de $38 \%$ en el ascenso y $36 \%$ en el descenso.

- Anchura de carril de 3,50 m.

- Arcén en el sentido ascendente $=2,50 \mathrm{~m}$

- Arcén en el sentido descendente $=1,50 \mathrm{~m}$

- Número de acceso por milla $=0$.

- Velocidad a flujo libre básica (BFFS): $106,2 \mathrm{~km} / \mathrm{h}$ en el ascenso y $118,8 \mathrm{~km} / \mathrm{h}$ en el descenso.

- $\quad$ Longitud del carril lento $=5822 \mathrm{~m}$

Se desarrolló el procedimiento del HCM para determinar el Nivel de Servicio de la carretera convencional sin carril lento arrojando los resultados de la Tabla 6. 


\begin{tabular}{|c|c|c|}
\hline INDICADOR & VALOR & NIVEL DE SERVICIO \\
\hline ATS $_{\mathrm{d}}$ & $97,2 \mathrm{~km} / \mathrm{h}$ & $\mathrm{A}$ \\
\hline PTSF $_{\mathrm{d}}$ & $51 \%$ & $\mathrm{C}$ \\
\hline
\end{tabular}

Tabla 6. Nivel de servicio en la carretera convencional según procedimiento del HCM (TRB, 2010).

Posteriormente se aplicó el procedimiento para estimar el nivel de servicio en la carretera convencional conteniendo el carril lento y considerando que el segmento de carretera CV13 está constituido solo por el carril lento con longitud $\left(\mathrm{L}_{\mathrm{pl}}\right)$ de $2630 \mathrm{~m}$, es decir, no se consideraron las regiones previas ni posteriores al carril lento: $\mathrm{L}_{\mathrm{u}}$, Longitud antes del carril adicional; $L_{d e}$, longitud efectiva y $L_{d}$, Longitud después de la longitud efectiva $L_{d e}$.

Se obtuvieron los resultados de la Tabla 7 que refleja la mejoría de la calidad de circulación en relación a la carretera sin carril lento.

\begin{tabular}{|c|c|c|}
\hline INDICADOR & VALOR & NIVEL DE SERVICIO \\
\hline $\mathrm{ATS}_{\mathrm{pl}}$ & $111 \mathrm{~km} / \mathrm{h}$ & $\mathrm{A}$ \\
\hline $\mathrm{PTSF}_{\mathrm{pl}}$ & $10 \%$ & $\mathrm{~A}$ \\
\hline
\end{tabular}

Tabla 7. Nivel de servicio en la carretera con carril lento según procedimiento del HCM (TRB, 2010).

\subsection{Nivel de servicio con TWOPAS calibrado}

Los resultados de aplicar el TWOPAS (TAM-IHSDM) calibrado en la carretera CV13 y considerando, en primera instancia, toda la carretera convencional (P.K. 3+880.500 y P.K.16+343,400) y luego solo el carril lento (P.K. 5+271.100 to 7+709.800) se presentan en las Tablas 8 y 9 , respectivamente.

\begin{tabular}{|c|c|c|c|c|c|c|c|c|c|c|}
\hline $\begin{array}{c}\text { Direction of } \\
\text { Travel }\end{array}$ & $\begin{array}{c}\text { Flow Rate from } \\
\text { Simulation } \\
(\mathrm{vph})\end{array}$ & $\begin{array}{c}\text { Percent Time } \\
\text { Spent } \\
\text { Following (\%) }\end{array}$ & $\begin{array}{l}\text { Average } \\
\text { Travel } \\
\text { Speed } \\
(\mathrm{km} / \mathrm{h}) \\
\end{array}$ & $\begin{array}{c}\text { Trip } \\
\text { Time } \\
(\mathrm{min} / \mathrm{veh})\end{array}$ & $\begin{array}{c}\text { Traffic } \\
\text { Delay } \\
(\mathrm{min} / \mathrm{veh})\end{array}$ & $\begin{array}{l}\text { Geometric } \\
\text { Delay } \\
\text { (min/veh) }\end{array}$ & $\begin{array}{c}\text { Total } \\
\text { Delay } \\
(\mathrm{min} / \mathrm{veh})\end{array}$ & $\begin{array}{l}\text { Number } \\
\text { of Passes }\end{array}$ & $\begin{array}{c}\text { Distance } \\
\text { Traveled } \\
(\mathrm{km})\end{array}$ & $\begin{array}{c}\text { Total } \\
\text { Travel } \\
\text { Time } \\
\text { (veh-hrs) }\end{array}$ \\
\hline Increasing & 167 & 44 & 87.7 & 8.5 & 0.3 & 1.0 & 1.3 & 47 & $2,065.6$ & 23.6 \\
\hline Decreasing & 181 & 40 & 65.5 & 11.2 & 0.8 & 3.3 & 4.1 & 447 & $2,217.2$ & 33.9 \\
\hline Combined & 348 & 42 & 76.2 & 9.9 & 0.6 & 2.2 & 2.8 & 494 & $4,282.8$ & 57.4 \\
\hline
\end{tabular}

Tabla 8. Resultados entre P.K. 3+880.500 y P.K.16+343,400.

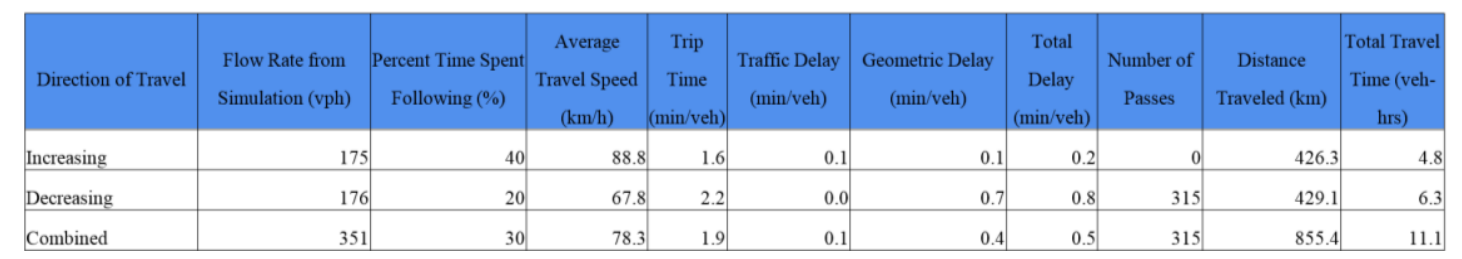

Tabla 9. Resultados entre P.K. 5+271.100 to 7+709.800 que contiene el carril lento. 
Los resultados generales se ilustran considerando varios indicadores a lo largo del recorrido de la carretera que se muestran en la Figura 3 solo para el sentido de circulación que contiene el carril lento. La graficas de dicha figura corresponden a:

- Ubicación de intersecciones.

- Altitud.

- Valor de $\mathrm{K}$ en $\mathrm{m} / \%$ para las curvas verticales.

- Grado de curvatura en grados sexagesimales de las curvas horizontales.

- Radio de las curvas horizontales en metros.

- Porcentaje de Tiempo consumido en el seguimiento vehicular (PTSF).

- Volumen en veh/h.

- Velocidad para autos y camiones en $\mathrm{km} / \mathrm{h}$.

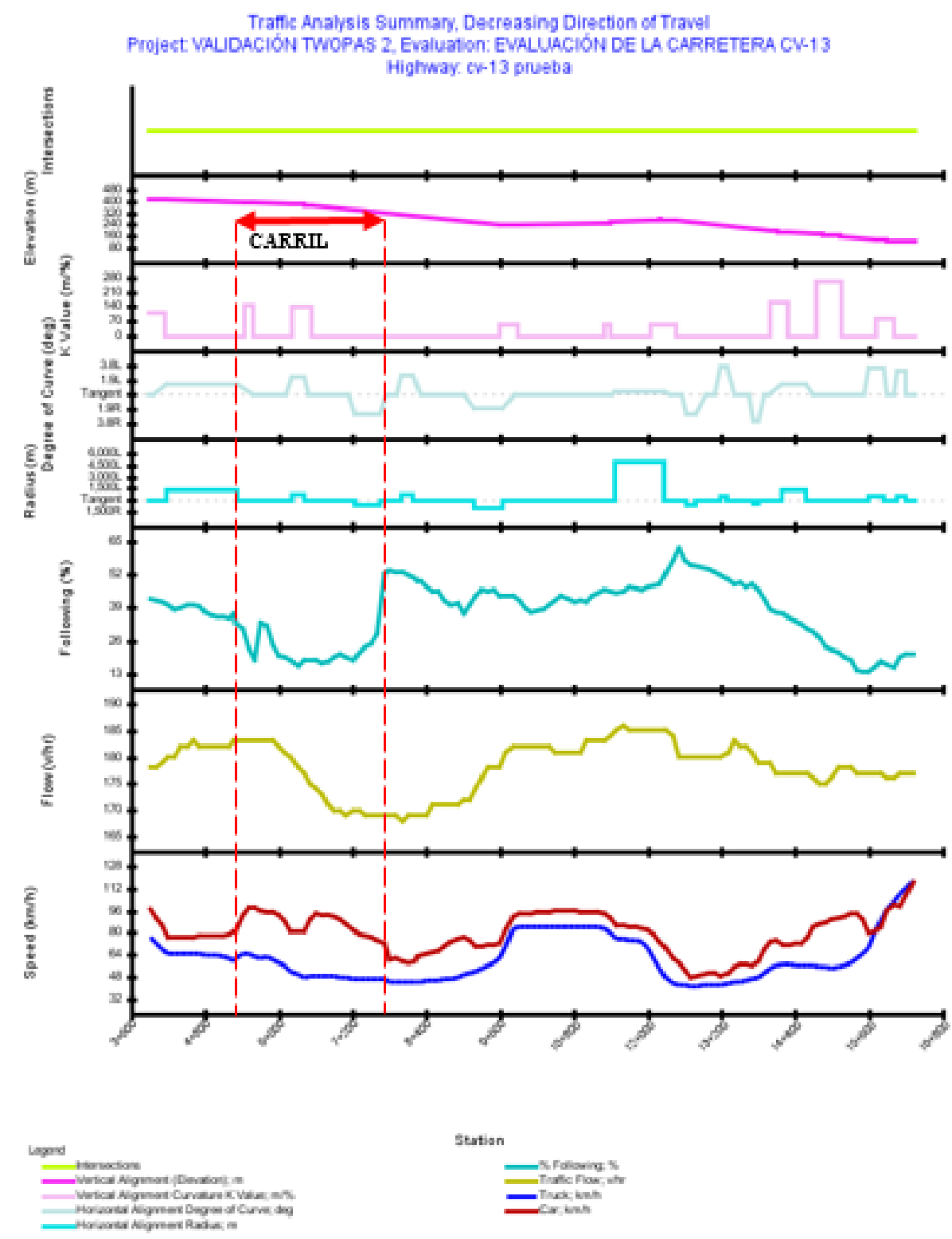

Figura 3. Resumen del análisis de tránsito en la CV-13 sentido decreciente de P.K. 
Según los resultados de PTSF y ATS en el segmento que contiene el carril lento de la Tabla 9 y considerando los criterios para el nivel de servicio en carreteras convencionales del HCM 2010 los niveles de servicio resultantes se muestran en la Tabla 10.

\begin{tabular}{|c|c|c|}
\hline TRAMO & INDICADOR & NIVEL DE SERVICIO \\
\hline Toda la carretera & ATS $=65,5 \mathrm{~km} / \mathrm{h}=40,9 \mathrm{mi} / \mathrm{h}$ & $\mathrm{D}$ \\
\hline & $\mathrm{PTSF}=40$ & $\mathrm{~B}$ \\
\hline Carril lento & $\mathrm{ATS}=67,8 \mathrm{~km} / \mathrm{h}=42,4 \mathrm{mi} / \mathrm{h}$ & $\mathrm{D}$ \\
\hline & $\mathrm{PTSF}=20$ & $\mathrm{~A}$ \\
\hline
\end{tabular}

Tabla 10. Nivel de servicio sin y con carril lento según simulación con TWOPAS.

\section{CONCLUSIONES}

- Las mediciones de relación peso/potencia utilizada en la circulación de los camiones arrojó como resultado un valor de $88 \mathrm{~kg} / \mathrm{CV}$ que corresponde, de manera práctica, con el vehículo de diseño considerado en el criterio del Libro Verde (AASHTO, 2011) de $120 \mathrm{~kg} / \mathrm{KW}(90 \mathrm{~kg} / \mathrm{CV}$ ) para justificar la dotación de carriles lentos y que ha sido acogido por la normativa española reciente evidenciando la correspondencia con la situación real observada en la vía.

- El grado de aproximación aceptable obtenido entre los indicadores simulados por el TWOPAS y los medidos en la CV13 permiten concluir que este modelo resulta calibrado para la carretera CV13 habilitándose como herramienta de análisis del tránsito así como fue usada en este trabajo.

- Según los resultados de calibración obtenidos puede considerarse que la calibración del TWOPAS puede ser mejorada abordando algunos procedimientos adicionales como medidas más detalladas de otros indicadores como las demoras geométricas, demoras por tránsito, número de adelantamientos y otros que son objeto de un estudio, continuación del presente, para estudiar la justificación de la conversión de carreteras convencionales a multicarriles.

- Al comparar los niveles de servicio resultantes considerando los dos procedimientos usados, el del HCM y usando los resultados de la simulación, conducen a concluir que ambos muestran mejoría en la operación vehicular con la provisión del carril lento principalmente con el indicador PTSF pero se mantiene con el ATS.

- A pesar que el TWOPAS fue calibrado, y por esto podría considerarse que los resultados de los indicadores representan el comportamiento del tránsito en la 
CV13, estos difieren de los resultados de los indicadores obtenidos por el procedimiento del HCM que considera el mismo vehículo patrón, aquel con relación peos/potencia de $90 \mathrm{~kg} / \mathrm{CV}$, principalmente en el indicador PTSF por lo que se concluye que es necesario mejorar la calibración del TWOPAS o adaptar el procedimiento del HCM a las condiciones de España.

- Según los resultados de la simulación de la CV13 con el TWOPAS y particularmente en la evaluación del carril lento se destaca la ubicación extraña del carril lento al final de la rampa cuando los indicadores de PTSF y ATS ilustrados en la Figura 3 indican que el mayor beneficio se hubiese logrado si este carril lento iniciara cerca del comienzo la rampa en el mismo sentido de circulación. El lugar más conveniente operacionalmente se puede determinar mediante una serie de aplicaciones del TWOPAS calibrado como lo recomendaron Valencia y García en 2010.

\section{REFERENCIAS}

- AASHTO. (2011). A Policy on Geometric Design of Highways and Streets, fifth edition. Washington, D. C.: AASHTO. Washington, D.C. EUA. xliv + 1006 pp

- Brewer, M. A. y Venglar, S. P. (2010). Passing behavior of drivers on Super 2 highways in Texas. En: 4th International Symposium on Highway Geometric Design. Valencia, Spain.

- FHWA. (2013). Traffic Analysis Module Engineer's Manual. Federal Highway Administration. Office of Safety Research and Development, Turner-Fairbank Highway Research Center, McLean, VA. September.

- Freedman, Z. and Al-Kaisy, A. (2013). Investigation of performance and lane utilization within a passing lane on a two lane rural highway. En: International Journal for Traffic and Transport Engineering, 2013, 3(3): 279 - 290.

- Generalitat Valenciana. (2010). Puesta en servicio de la CV-13 entre la CV10 (Benlloch) y la AP7 (Torreblanca). Conselleria D’Infraestructures I Transport. Valencia (España). (Valencia A., V. G. (1998 a) Estudio de rampas en carreteras convencionales para la optimización del alineamiento y sus efectos operacionales, ambientales y económicos. Tesina de especialización. Universidad Politécnica de Valencia. Universidad Pedagógica y Tecnológica de Colombia. España.)

- GLENNON, J. C. (1970). An Evaluation of Design Criteria for Overtaking Trucks Safely on Grades. Highway Research Record 312, Highway Research Board. 93$112 \mathrm{pp}$.

- Harwood, D. W. \& St. John, A. D. (1986). Operational effectiveness of passing lanes on two-lane highways. Report No FHWA/RD-86/196. Federal Highway Administration. April. 
- Texas Transportation Institute, The Texas A\&M University (2001) Design Guidelines for passing lanes on two-lane roadways (Super 2). FHWA report 40611. Texas Department of Transportation Research and Technology Implementation Office, Austin, Texas pp 106.

- Ahmed, Al-Kaisy and Zachary, Freedman. (2010). Empirical Examination of Passing Lane Operational Benefits on Rural Two Lane Highways. En: Journal of the Transportation Research Forum, Vol. 49, No. 3 (Fall 2010), pp. 53-68.

- Khan, A. M. et al. (1990). Heavy Vehicle Performance on Grade and Climbing Lane Criteria. Research and Development Branch, Ministry of Transportation. Ontario, Canadá. Noviembre, pp. x +78 .

- Khan, A. M. et al. (1991). Cost-effectiveness of Passing Lanes: Safety, Level of Service, and Cost Factors. Research and Development Branch, Ministry of Transportation. Ontario, Canadá. September, pp. xi +124.

- May, A. D. (1991). Traffic Performance and Design of Passing Lanes. In Transportation Research Record 1303, TRB, National Research Council, Washington, D. C., pp. 63-73.

- Messer C. J., (1983). Two-lane, two-way highway capacity. Final report of NCHRP Project 3-28A. Transportation Research Board.

- MINISTERIO DE FOMENTO DE ESPAÑA (2016). Norma 3.1-IC. Trazado (Orden FOM/273/2016 de 19 de febrero de 2016). Consulta en Internet: http://www.fomento.gob.es/MFOM/LANG_CASTELLANO/DIRECCIONES GENERALES/CARRETERAS/NORMATIVA_TECNICA/TRAZADO/.

- Morrall, J. y Thomson, W. (1990). Planning and Design of Passing Lanes for the Trans-Canada Highways in Yoho National Park. En Canada Journal of Civil Engineering, Vol. 17, № 1. Canadá, Febrero pp. 79-86.

- NCHRP (1998). Capacity and quality of service of two-lane highways. NCHRP PROJECT 3-55(3). Task 6, Enhance, calibrate, and validate the selected simulation model. TWOPAS model improvements.

- Transportation Research Board (2010). Highway Capacity Manual. HCM 2010. Washington, D. C.: Transportation Research Board. E.U.

- Valencia, V. G., Bedoya, V. E. y Osorno, M. E. (1996) Relación Peso/Potencia de Vehículos Pesados en Colombia. En: Memorias del IX Congreso Panamericano de Ingeniería de Tránsito y Transporte. Diciembre 6. La Habana (Cuba).

- Valencia, V. y García, A. (2010). Procedures to Facilitate Passing on Conventional Highways by Means of Simulation. En: Proceedings of 4th International Symposium on Highway Geometric Design. Valencia (España). Junio 1 al 5.

- Valencia Alaix, V. G. (2016). Elaboración de procedimientos para facilitar el adelantamiento en carreteras convencionales aplicando simulación [Tesis doctoral no publicada]. Universitat Politècnica de València. doi:10.4995/Thesis/10251/62413. 